\title{
Integration of water resources modelling approaches for varying levels of decision-making
}

\author{
S. J. L. Mallory ${ }^{1} \&$ S. J. van Vuuren ${ }^{2}$ \\ ${ }^{1}$ Water for Africa (Pty) Ltd, South Africa \\ ${ }^{2}$ University of Pretoria, South Africa
}

\begin{abstract}
Within government organisations there is a hierarchy of decision making ranging from broad, strategic decisions taken at top management level, through detailed planning, to the routine operational decisions. Due to the complex nature of water resources management, some form of water resources model is required to determine how much water is available, and to balance this against water requirements, taking the variability in assurance of supply to different users into consideration. In South Africa, as in many other countries, the trend has been to develop different models aimed at advising different levels of decision making. While this has proved successful, it has led to multiple models and hence multiple databases, and inevitably to inconsistencies in the conclusions reached by adopting different scales of modelling intensity and complexity. This paper describes an approach to water resources modelling which seamlessly deals with several levels of complexity, from broad-scale, strategic planning through detailed planning (or systems analysis), to the short to medium term operation of reservoirs (including hedging strategies) to deal with droughts and unexpected situations such as over-abstraction. The initial model setup commences at a broad strategic (or reconnaissance) level. As the model user progresses to higher levels of modelling intensity he is prompted to provide the data required by these higher levels. The core of this modelling system is a database of water user and monthly naturalized hydrology, rainfall and evaporation which is used throughout all levels of modelling. The fundamental concepts of reservoir behaviour analysis forms the basis of the modelling procedure, commencing with a single iteration cascading monthly time-step simulation for strategic planning purposes and progressing to a multi-iteration solution using up to 500 stochastically generated hydrological sequences, including curtailment rules for each user, to solve complex reservoir operation problems. Comprehensive testing and application of this system has not yet been undertaken, but preliminary observations are made in this paper based on a trial application in a test catchment.
\end{abstract}

Keywords: $\quad$ water resources modelling, reservoir simulation, database structures, reconnaissance modelling, systems analysis, reservoir operation. 


\section{Introduction}

The trend in water resources model development today seems to be towards the optimization of increasingly complex systems. Optimization of systems in developed countries is undoubtedly necessary as available water resources become increasingly stressed. However, in developing countries it is important to first gain a fundamental understanding of the water resources of the country or region as a whole before embarking on detailed and costly optimisation analyses. This broad or strategic level of understanding is needed by high level decision makers who are, however, unlikely to have the required expertise in water resources modelling. Simpler models are therefore required to support this strategic level of understanding.

A frequently overlooked but important aspect of modelling is that when model developers strive to solve increasingly complex problems, their models tend to become correspondingly more difficult to understand and use, often leaving the decision maker behind in the process. There is therefore a niche to be filled by simpler models, which may be less accurate and unable to deal with complex problems, but which can at least provide a broad level of understanding to the decision maker at a higher level of management within an organization.

There is, therefore, a trade off between simple, easy to use models, which do not adequately address the complexities of the actual operation of a catchment, and the more complex models capable of addressing these issues but which are not accessible to higher levels of management or decision makers.

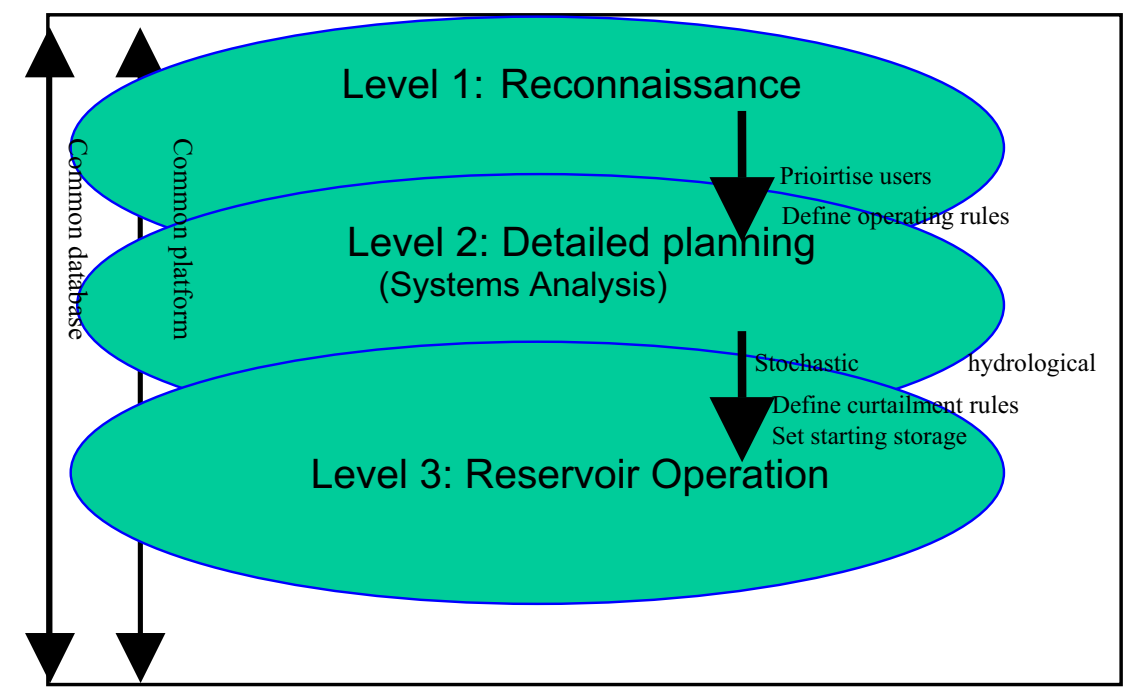

Figure 1: Hierarchical modeling: conceptual layout. 
This paper discusses the integration of modelling approaches at various levels (Reconnaissance, Systems Analysis, and short-term Reservoir Operation) onto a single platform in order to allow organizations (or individuals) to progress seamlessly from simple strategic modelling through to more complex operational modelling using the same underlying data and model structure. The objective of such a modelling platform is to improve efficiency. Training and support costs could be substantially reduced through the use of such a system, since many of the supporting tools such as graphics and GIS interfaces are the same regardless of the level at which the model is being used. This hierarchical modelling concept is presented in Figure 1.

\section{Principles of deploying and managing a common database}

An obvious but often neglected prerequisite to achieving consistent and defendable decision-making, from the broad strategic level through to detailed operation of complex systems, is the use of a common data source for both the hydrology and the water use data within the region under consideration. Furthermore, in order to achieve the objective of reconnaissance level or strategic planning, models must be sufficiently flexible, and able to function rapidly enough to run in a workshop environment. It is argued that this can only be achieved by setting up a sub-catchment reference system in which all the required information is referenced to the sub-catchments in which they occur. It is also essential to use the same sub-catchment reference system for all levels of modelling if the results are to be comparable as increasing levels of modelling sophistication are added.

In South Africa, a standard sub-catchment reference system, referred to as quaternary catchments, was established in the early 1990's. (Midgely et al [1]). This data set, which consists of monthly streamflow and rainfall time series as well as mean monthly evaporation data are readily available in Midgely's suite of reports as well as on the SPATSIM database (Hughes [2]). Other South African databases, such as the Information Management System (IMS) (Nyland and Watson [3]) or the WSAM (Schulze and Watson [4]) either do not use the accepted catchment definition as described in Midgely et al [1] or else only contain annual mean data rather than the entire time series. In the case of the modeling system described in this paper, referred to as the Water Resources Modelling Platform (WReMP), Midgely's quaternary catchment names are used a reference to create a simple Paradox database in order to make it readily available for modelling. Simply by referencing the sub-catchment name, the natural flow and rainfall time series data are loaded into the model at run time. Spatially referenced data such as this also lends itself to easy deployment on GIS, an important feature in any water resources model.

Water use data is more difficult to manage than hydrological data since it is constantly subject to change. Water use data was collected in South Africa for the whole country in preparation for the development of the National Water Resources Strategy (NWRS) (Department of Water Affairs and Forestry [5]) and is readily available as mean annual values through the WSAM model 
(Schulze and Watson [4]). However, a mechanism to manage and update this data, together with the monthly distribution of these water demands is still lacking. This is important for efficient modelling and has been dealt with in the development of this modelling platform through the concept of data mapping. Data mapping entails the creation of a sub-set of a National database for each scenario to be modelled. As a default operation, the relevant quaternary catchment data is copied from the National database into the sub-set database which can then be edited and saved by the user without over-writing (and hence corrupting) the National database.

The objective of structuring these databases was to strive for the minimum amount of data which is required by a monthly time step model. Water demands that are largely independent of rainfall events can be described adequately by the annual average demand in each sub-catchment as well as by twelve monthly factors which distribute the annual requirement realistically into twelve monthly values. The water demands have been categorized into user sectors, namely, rural, strategic, industrial, mining, urban and irrigation. Due to the limited water resources in South Africa, the priority and assurance of supply differs across user sectors. An important outcome of any water resources modeling is a strategy or operating rule which ensures that the various water use sectors will receive their water at the levels of assurance specified in the NWRS. These user sectors are therefore modeled separately so as to give a sectoral breakdown of water requirements and supply as required by the NWRS.

The water requirements of the irrigation sector, as well as those for the ecology are highly dependant on rainfall events. A separate irrigation database is used which contains crop areas and crop factors for each sub-catchment, again copied from a National database. Crop requirements are then calculated at each time step taking account of the estimated rainfall in each month. Ecological water requirements (EWR) are determined on a month by month basis using a pre-defined relationship between the natural flow and the EWR, which is provided by ecological specialists. Currently there is no complete database of the EWRs in South Africa, which is a limitation on the application of the modeling techniques described in this paper. The data that is available is stored as text files, which are accessed by the model via the catchment reference name.

\section{Level 1: Reconnaissance level}

The reconnaissance level modeling proposed by this paper does not read water use data from a time series file but rather minimizes user input by calculating it at every time step using the annual average requirement and the monthly distribution information provided by the water use database. See Eqn (1). Water requirements are calculated in order of priority and then checked to see how much of the requirement can actually be supplied either from storage or from the available flow in the river, before proceeding to the next user sector.

$$
\text { Requirement }[j, i, t]=(A A R[j, i])(D F[j, i, m n t h])
$$


where: Requirement $[j, i, t]=$ Water requirement of user $j$ and node $i$ at time step $t$

$A A R[j, i]=$ Average annual requirement of user $j$ at node $i$.

$D F[j, i, m n t h]=$ Monthly distribution factor for user $j$ and node $i$ for month mnth.

Should the situation occur, where there is insufficient storage or flow to meet the requirement of user $\mathrm{j}$ then all users of lesser priority, i.e. $j+1, j+2$, etc, will not receive any water. In a well managed system, users would be restricted in times of drought with the aim of supplying all users with some water, a strategy which is modeled in more detail in the Reservoir Operation mode. Reconnaissance mode however makes the simplifying assumption of pre-defined prioritization of use at each node.

The result of the simplification presented in eqn (1) is that while there is monthly variation in the water requirement, the requirement is constant from one year to the next. The actual supply can however vary since if water is not available the supply will be less than the requirement.

Irrigation requirements are calculated every month during model run time using a typical crop requirement equation which takes into account effective rainfall and the efficiency of the irrigation method used. For brevity, these equations are not repeated here. The reader is referred to website of the Food and Agricultural Organisation [6] for more details on this aspect.

The output from a reconnaissance level simulation is a summary of all requirements and actual supply, expressed as annual average values as well as a calculation of the yield that is theoretically obtainable from each sub-catchment, also referred to as a node, within the model.

Yields are expressed in the following forms, as described in detail in Mallory [7].

- Cumulative yield expresses the yield that could be obtained at a particular node given all inflows (and hence taking into account all upstream abstractions and accumulating all inflows up to the node) but not taking into account abstraction from the node under consideration.

- Incremental yield expresses the yield that could be obtained if the calculated upstream yield was abstracted. The concept of incremental yield is useful in that it gives a good indication of the relative ability of each sub-catchment in a system to generate utilizable yield, without clouding the issue of what abstractions are already taking place within the catchment. Incremental yields can also be summed, to give an indication of the total yield available in a catchment if operated in a simple cascading fashion.

- The yield balance indicates the yield remaining at each node in the system after all abstractions at the node. This is a useful indicator of catchment 
stress: if the yield balance is zero, it is very likely that not all the water demands at the node can be met.

\section{Level 2: Systems analysis modelling (for detailed planning)}

The reconnaissance level modelling proposed above assumes a cascading priority of water use in a catchment, which is not necessarily always the case. The sectoral water supply priorities defined in South Africa's NWRS are not related in any way to a user's location within a catchment. Hence, a high-priority user at the downstream end of a catchment poses a challenge, both in terms of the actual operation of the catchment as well as the modelling of this operation. Numerous models are available which deal with these complexities, popular methods for solving such systems numerically being linear programming and dynamic programming. South Africa's Water Resources Yield Model (WRYM) is based on the Canadian ACRES model which uses the out-of-kilter algorithm essentially a form of linear programming (Department of Water Affairs and Forestry [8]).

The modelling system proposed in this paper for Level 2 modelling or systems analysis uses an iterative cascading solution similar to that used in the HEC5 model (Hydrological Engineering Centre [9]). It is described in more detail in Mallory and van Vuuren [10]. The shortages or deficits experienced by high priority downstream users are calculated in the first iteration. In the second iteration the shortages are released from storage or allowed to flow past upstream users by imposing curtailment rules. This method closely duplicates the actual method used by catchment managers to meet high priority downstream demands. This is an advantage over linear programming methodologies, which are based on subjective weighting factors or penalties, which are then minimized by the model, without necessarily providing any insights as to how the catchment is actually operated. Determining operating rules for a large number of users can however be time-consuming in stressed catchments using curtailment rules. Until such time as this process can be automated there is still a place in water resources modelling for linear programming models.

The advantage of the iterative cascading solution described in Mallory and van Vuuren [10] is that it is a simple extension of the proposed reconnaissance level model described above. To advance the reconnaissance level model to systems analysis mode requires the following input from the modeler:

Multiple sources of water supply: In most water supply systems, water users can obtain their water from more than one source although they may not even be aware of this. For example, an irrigation scheme typically makes use of run-ofriver flow as its principal source of water, but when required, this run of river flow is supplemented by releases from upstream dams. In the Systems Analysis mode described here this would be defined as multiple source of supply, with the priority source being the run-of-river flow.

Curtailment rules: In catchments where no dams are available to supply downstream users, the catchment manager could impose water restrictions on 
upstream users in order to ensure that water earmarked for high-priority downstream users does actually reach them. These restrictions can either be described as a function of actual river flow, or natural flow, or the water level in a reservoir. Currently within WReMP, these curtailment rules are determined through trial and error which can be a time-consuming and inefficient exercise in large, water-stressed catchments.

User-defined time series: While the reconnaissance level model makes use of readily available information through an indexed database, the modeler may have access to better, more detailed information. By opting for time series input, the modeler can use other models to generate these time series if he so wishes or use time series provided by others with expertise in specific fields such as irrigation. The Systems Analysis mode therefore switches from "on-the-fly" calculation of demands as defined by eqn (1) to user-defined time series of all water demands. Utilities are also provided within the WReMP modelling framework to generate these time series from the database.

Return flows: The systems analysis mode allows the user to specify the node at which return flows accumulate, as well as the percentage of the flow that is returned.

Stochastic hydrological sequences: Stochastic analysis is introduced at the Systems Analysis level of modelling, allowing the modeler to source stochastic hydrological sequences from his preferred stochastic hydrological model. WReMP allows up to 500 hydrological sequences for every node in the system.

Advancing to Systems Analysis mode, the following additional output is ouput is provided:

- time series plots of releases made from dams to meet downstream demands.

- $\quad$ So called long-term yield curves (Basson et al [11]). These curves are useful to estimate the degree of reliability associated with the historic yield.

\section{Level 3: Reservoir operation modelling}

The objective of Reservoir Operation mode is to ensure that users obtain some water all of the time rather than all of their water for some of the time. In arid countries such as South Africa, which have highly seasonal and erratic rainfall, it is common practice to progressively curtail abstractions from reservoirs as the storage is progressively depleted. This type of operation, also referred to as hedging, may seem to indicate a lack of faith in the planning process which has already determined the yield that can be obtained from a dam, as well as the level of assurance of that supply. The reality is, however, that hydrology is not an exact science and there is no guarantee that the next drought will not be worse than all the previous droughts that were used as the basis of planning.

The strategic level water resources planning that has been carried out in South Africa (Department of Water Affairs and Forestry [5]) is based on a drought recurrence interval of 1 in 50 years, which implies a $2 \%$ risk of the water supply system failing in any one year. When it comes to the actual operation of a bulk water supply system, the objective is that it must never fail. This is achieved by 
curtailing the water use as the reservoir progressively empties. Therefore, while such curtailments actually reduce the water supplied from the yield determined in Systems Analysis mode, the assurance of supply (or at least partial supply) increases to $100 \%$.

In order to model this type of reservoir operation, a curtailment rule is required for every source of supply to every user. The data structure for this is already provided in the Systems Analysis mode, since it is necessary to curtail users in line with the defined priorities. The additional information required to advance to Reserve Operation mode is as follows:

Curtailment rules: Required for each user abstracting from each reservoir. Currently these are established through trial and error until a scenario is reached in which the reservoirs do not fail.

Starting storage: This enables the modeller to take into account the actual storage in each reservoir in the system when making decision relating to curtailments.

Analysis length: This must be specified in years and should be a year or two longer than the critical period of the reservoir.

Number of stochastic sequences: As the number of hydrological sequences used in the model is increased, the probability of failure occurring in practice decreases provided that the curtailment or operating rule is adhered to. However, with increasing number of sequences, the model run time increases and hence a balance between accuracy and modelled intensity needs to be found.

The output from the Reservoir Operation mode of simulation would typically be a probabilistic plot of storage in the reservoir over time.

\section{Application of hierarchical modelling to a trial catchment}

The three modelling modes described in this paper have been applied individually to numerous systems in South Africa, notably during the development of the so-called Internal Strategic Perspectives (Department of Water Affairs and Forestry [12]), the Algoa Systems Analysis (in progress) and the modelling of the Letaba System (Department of Water Affairs and Forestry [13]). However, the concept of progressing seamlessly from Reconnaissance mode through to Reservoir Operation mode still needs to be thoroughly tested and documented. A trial run carried out in South Africa's Mgeni System, (which is Durban's source of water), has been carried out but space limitations of this publication prohibit detailed publication of the results of this trial run. Preliminary conclusions from this analysis are, however, as follows:

Reconnaissance level modelling tends to underestimate the yield of the system as a whole. This is not surprising since it is well documented (McMahon et al [14], Ndiritu [15]) that conjunctive use of reservoirs, and the conjunctive use of run-of-river flow and reservoirs can substantially increase system yield. 
Systems Analysis over-estimates assurance of supply when compared with the results of the Reservoir Operation mode. Systems Analysis is also a poor indicator of when the yield of a system needs to be augmented.

Reservoir Operation seems to be the most realistic modelling mode and should be used more extensively for important decision-making such as the allocation of water to users in stressed systems and in determining time frames for augmentation of the supply system. This operation mode is however the most complex and time consuming.

\section{Conclusions}

A hierarchical water resource modelling system has been developed which enables modelers or organisations to progress easily from the simple modeling required for large catchments to make broad strategic decisions, through to the detailed modeling of reservoir operations and curtailment of water supply to users in response to drought conditions. This modeling system, referred to as the Water Resources Modelling Platform, offers efficiency through the use of a common database of hydrological and water use information which is used at all levels of complexity, as well as through the use of a common interface. The main advantage of such a system is that it allows decision makers to make reasoned decisions backed by scientifically sound analysis as to which water supply systems should be advanced to higher levels of modeling detail and intensity.

While the individual modeling modes described in this paper have been thoroughly tested and applied in practice on numerous projects, thorough testing and application of the integrated system is still required.

\section{References}

[1] Midgley, D C, Pitman, W V and Middleton, B J. Surface Water Resources of South Africa 1990. Appendices and Book of Maps. WRC Report Number 298/6.1/94. 1994.

[2] Hughes, DA. SPATSIM, an integrating framework for ecological Reserve determination and implementation. Water Research Commission Report No. TT 245/04. 2004.

[3] Nyland G F, Watson M D. Water Resources Decision Support Framework (WRDSF). 12th South African National Hydrology Symposium. 2005.

[4] Schulze C, Watson M D. Water Situation Assessment Model - A decision support system for reconnaissance level planning. Theoretical Guide compiled for the Department of Water Affairs and Forestry. 2002.2.

[5] Department of Water Affairs and Forestry, South Africa. National Water Resource Strategy, First Edition. 2004.

[6] Food and Agricultural Organisation. www.FOA.org.za.

[7] Mallory, SJL. Water resources modelling for the National Water Act. Eleventh South African National Hydrology Symposium. 2003.

[8] Department of Water Affairs and Forestry, South Africa. Water Resources Yield Model User Guide. Report no. P0000/00/001/98. 1998. 
[9] Hydrological EngineeringCentre, US Army Corps of Engineers. HEC-5: Simulation of flood control and conservation systems: User Manual. 1989.

[10] Mallory S J L, van Vuuren S J. A practical approach to water resources modelling of complex catchments. In Press.

[11] Basson M S, Allen R B, Pegram G G S, van Rooyen J A. Probabilistic Management of Water Resource and Hydropower Systems. Water Resources Publications, Colorado, USA. 1994.

[12] Department of Water Affairs and Forestry, South Africa. Internal Strategic Perspective: Mvoti to Mzimkulu Water Management Area. Report no. P WMA 11/000/00/0304. 2004.

[13] Department of Water Affairs and Forestry, South Africa; River System Annual Operating Analysis (2005/2006), 2006. Report no. P WMA 02/000/00/0406. 2006.

[14] McMahon, T and Adeloye, A. Water Resources Yield. WRP Publications, Colorado, USA. 12 - 80. 2005.

[15] Ndiritu J G. Maximising water supply system yield subject to multiple reliability constraints via simulation-optimisation. Water SA 31(4) $423-$ 434. 2005. 